PROCEEDINGS OF THE

AMERICAN MATHEMATICAL SOCIETY

Volume 136, Number 6, June 2008, Pages 2201-2207

S 0002-9939(08)09230-7

Article electronically published on February 12, 2008

\title{
UNIQUENESS OF THE SOLUTION OF A PARTIAL DIFFERENTIAL EQUATION PROBLEM WITH A NON-CONSTANT COEFFICIENT
}

\author{
ERNESTO PRADO LOPES AND JOSÉ ROBERTO LINHARES DE MATTOS
}

(Communicated by David S. Tartakoff)

\begin{abstract}
We consider the problem $K(x) u_{x x}=u_{t}, 0<x<1, t \geq 0$, where $K(x)$ is bounded below by a positive constant. The solution on the boundary $x=0$ is a known function and $u_{x}(0, t)=0$. This is an ill-posed problem in the sense that a small disturbance on the boundary specification can produce a big change in its solution, if it exists. In a previous work, we used a Wavelet Galerkin Method with the Meyer Multiresolution Analysis to generate a sequence of well-posed approximating problems to it. In the present work, by assuming that $1 / K(x)$ is Lipschitz, we are able to prove that the existence of a solution $u(x, \cdot) \in H^{1}(R)$, for this problem, implies its uniqueness.
\end{abstract}

\section{INTRODUCTION}

We consider the following problem for $0<\alpha \leq K(x)<+\infty$ :

$$
\begin{cases}K(x) u_{x x}(x, t)=u_{t}(x, t), & t \geq 0, \\ u(0, \cdot)=g, & u_{x}(0, \cdot)=0 .\end{cases}
$$

We assume $K$ to be continuous, $g \in L^{2}(R)$ when it is extended as vanishing for $t<0$, and the problem to have a solution $u(x, \cdot) \in H^{1}(R)$ when it is extended as vanishing for $t<0$.

Problem (1.1) is ill-posed in the sense that a small disturbance on the boundary specification $g$ can produce a big change in its solution, if it exists. This means that if the solution exists, it does not depend continuously on $g$ (see [2, page 224]).

We consider the Meyer Multiresolution Analysis. The advantage in making use of Meyer's wavelets is its good localization in the frequency domain, since its Fourier transform has compact support. Orthogonal projections onto Meyer's scaling spaces can be considered as low pass filters, cutting off the high frequencies.

From the variational formulation of the approximating problem in the scaling space $V_{j}$, we get an infinite-dimensional system of second-order ordinary differential equations with variable coefficients. The ill-posed problem is regularized by approaching it by well-posed problems (see Theorem 3.4 in [2, page 221]).

We consider that $1 / K(x)$ is Lipschitz and we prove that the existence of a solution $u(x, \cdot) \in H^{1}(R)$ implies its uniqueness.

Received by the editors October 18, 2006.

2000 Mathematics Subject Classification. Primary 65T60.

(C)2008 American Mathematical Society 
We would like to point out that our result is weaker than the overall uniqueness of a solution $u(\cdot, \cdot)$ of problem (1.1), which cannot be discussed without further conditions on this problem. Our uniqueness result supposes that $x \in(0,1)$ is fixed, and it is the solution $u(x, \cdot) \in H^{1}(R)$, as function of the second variable, which is proved to be unique. More precisely, a solution $u(x, \cdot)$ can only be modified in a subset of $[0,+\infty)$ of measure zero.

The Fourier Transform of a function $h \in L^{1}(R) \cap L^{2}(R)$ is given by $\widehat{h}(\xi):=$ $\int_{R} h(x) e^{-i x \xi} d x$. We use the notation $e^{x}$ and $\exp x$ indistinctly.

\section{UNIQUENESS}

A multiresolution analysis, as defined in [1], is a sequence of closed subspaces $V_{j}$ in $L^{2}(R)$, called scaling spaces, satisfying:

(M1) $V_{j} \subseteq V_{j-1}$ for all $j \in Z$.

(M2) $\bigcup_{j \in Z} V_{j}$ is dense in $L^{2}(R)$.

(M3) $\bigcap_{j \in Z} V_{j}=\{0\}$.

(M4) $f \in V_{j}$ if and only if $f\left(2^{j}\right.$. $) \in V_{0}$.

(M5) $f \in V_{0}$ if and only if $f(\cdot-k) \in V_{0}$ for all $k \in Z$.

(M6) There exists $\phi \in V_{0}$ such that $\left\{\phi_{0, k}: k \in Z\right\}$ is an orthonormal basis in $V_{0}$, where $\phi_{j, k}(x)=2^{-j / 2} \phi\left(2^{-j} x-k\right)$ for all $j, k \in Z$. The function $\phi$ is called the scaling function of the multiresolution analysis.

The scaling function of the Meyer Multiresolution Analysis is the function $\varphi$ defined by its Fourier Transform:

$$
\widehat{\varphi}(\xi):= \begin{cases}1, & |\xi| \leq \frac{2 \pi}{3} \\ \cos \left[\frac{\pi}{2} \nu\left(\frac{3}{2 \pi}|\xi|-1\right)\right], & \frac{2 \pi}{3} \leq|\xi| \leq \frac{4 \pi}{3} \\ 0, & |\xi|>\frac{4 \pi}{3}\end{cases}
$$

where $\nu$ is a diferentiable function satisfying

$$
\nu(x)=\left\{\begin{array}{lll}
0 & \text { if } & x \leq 0 \\
1 & \text { if } & x \geq 1
\end{array}\right.
$$

and

$$
\nu(x)+\nu(1-x)=1 .
$$

The associated mother wavelet $\psi$, called Meyer's Wavelet, is given by (see [1]

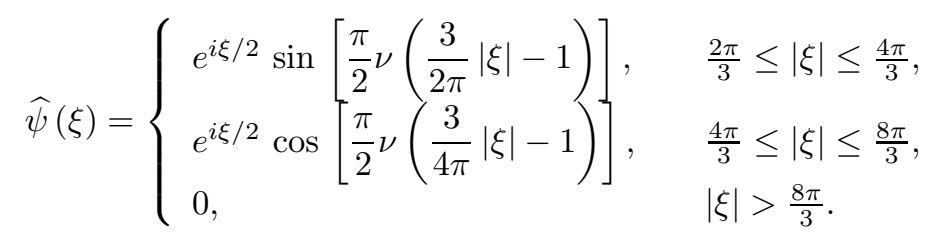

We will consider the Meyer Multiresolution Analysis with scaling function $\varphi$. The orthogonal projection onto $V_{j}, P_{j}: L^{2}(R) \rightarrow V_{j}$ is given by

$$
P_{j} f(t)=\sum_{k \in \mathbb{Z}}\left\langle f, \varphi_{j k}\right\rangle \varphi_{j k}(t)
$$

Lemma 1. The operator $D_{j}(x)$ defined by

$$
\left[\left(D_{j}\right)_{l k}(x)\right]_{l \in Z, k \in Z}=\left[\frac{1}{K(x)}\left\langle\varphi_{j l}^{\prime}, \varphi_{j k}\right\rangle\right]_{l \in Z, k \in Z}
$$


satisfies:

1) $\left(D_{j}\right)_{l k}(x)=-\left(D_{j}\right)_{k l}(x)$.

2) $\left(D_{j}\right)_{l k}(x)=\left(D_{j}\right)_{(l-k) 0}(x)$. Hence, $\left(D_{j}\right)_{l k}(x)$ is a Töplitz matrix.

3) $\left\|D_{j}(x)\right\| \leq \frac{\pi^{2} 2^{-j+1}}{K(x)}$.

Proof. 11) Since $\varphi$ is real and $\varphi_{j k}(x) \rightarrow 0$, when $x \rightarrow \pm \infty$, an integration by parts gives the result.

2) The substitution $2^{-j} s=2^{-j} t-k$ applied to $\left(D_{j}\right)_{l k}(x)$ gives:

$$
\begin{aligned}
\left(D_{j}\right)_{l k}(x) & =\frac{1}{K(x)} \int_{\mathbb{R}} \varphi_{j l}^{\prime}(t) \varphi_{j k}(t) d t=\frac{1}{K(x)} \int_{\mathbb{R}} \varphi_{j(l-k)}^{\prime}(s) \varphi_{j 0}(s) d s \\
& =\left(D_{j}\right)_{(l-k) 0}(x) .
\end{aligned}
$$

3) We have

$$
\left\|D_{j}(x)\right\|=\left\|\frac{1}{K(x)} B_{j}\right\|=\frac{1}{K(x)}\left\|B_{j}\right\|
$$

where $\left(B_{j}\right)_{l k}=\left\langle\varphi_{j l}^{\prime}, \varphi_{j k}\right\rangle$. From results 1) and 2), we have $\left(B_{j}\right)_{l k}=-\left(B_{j}\right)_{k l}$, $\left(B_{j}\right)_{l k}=\frac{1}{2 \pi} \int_{\mathbb{R}} \xi e^{-i(l-k) \xi 2^{j}}\left|\widehat{\varphi_{j 0}}(\xi)\right|^{2} d \xi=\left(B_{j}\right)_{(l-k) 0}$ and $\left(B_{j}\right)_{l k}$ is a Töplitz matrix. We will show that $\left\|B_{j}\right\| \leq \pi^{2} 2^{-j+1}$. Thus, we will have

$$
\left\|D_{j}(x)\right\| \leq \frac{\pi^{2}}{K(x)} 2^{-j+1} .
$$

For $|t| \leq \pi 2^{-j}$, let

$$
\begin{aligned}
\Gamma_{j}(t)= & i 2^{-j}\left[\left(t-2^{-j+1} \pi\right)\left|\widehat{\varphi_{j 0}}\left(t-2^{-j+1} \pi\right)\right|^{2}+t\left|\widehat{\varphi_{j 0}}(t)\right|^{2}\right. \\
& \left.+\left(t+2^{-j+1} \pi\right)\left|\widehat{\varphi_{j 0}}\left(t+2^{-j+1} \pi\right)\right|^{2}\right] .
\end{aligned}
$$

Extend $\Gamma_{j}$ periodically to $\mathbb{R}$ and expand it in a Fourier series as

$$
\Gamma_{j}(t)=\sum_{k \in \mathbb{Z}} \gamma_{k} e^{i k t 2^{j}}
$$

We have $\gamma_{k}=b_{k}$ for all $k$, where $b_{k}$ is the element in diagonal $k$ of $B_{j}$. In fact, since $\widehat{\varphi_{j 0}}(t)=0$ for $|t| \geq \frac{4}{3} \pi 2^{-j}$, it follows that

$$
\begin{aligned}
\gamma_{k}= & \frac{1}{2^{-j+1} \pi} \int_{-\pi 2^{-j}}^{\pi 2^{-j}} \Gamma_{j}(t) e^{-i k t 2^{j}} d t \\
= & \frac{i}{2 \pi} \int_{-\pi 2^{-j}}^{\pi 2^{-j}}\left(t-2^{-j+1} \pi\right)\left|\widehat{\varphi_{j 0}}\left(t-2^{-j+1} \pi\right)\right|^{2} e^{-i k t 2^{j}} d t \\
& +\frac{i}{2 \pi} \int_{-\pi 2^{-j}}^{\pi 2^{-j}} t\left|\widehat{\varphi_{j 0}}(t)\right|^{2} e^{-i k t 2^{j}} d t \\
& +\frac{i}{2 \pi} \int_{-\pi 2^{-j}}^{\pi 2^{-j}}\left(t+2^{-j+1} \pi\right)\left|\widehat{\varphi_{j 0}}\left(t+2^{-j+1} \pi\right)\right|^{2} e^{-i k t 2^{j}} d t .
\end{aligned}
$$

\footnotetext{
${ }^{1}$ We are grateful to Professor Rémi Vaillancourt, who pointed out some mistakes in part 3) of this lemma in our previous paper [2, page 216], and to the referee, who proposed a shorter proof for parts one and two. The present proof was revised in accordance with their suggestions.
} 
Making a change of variable, we obtain

$$
\begin{aligned}
\gamma_{k}= & \frac{i}{2 \pi} \int_{-3 \pi 2^{-j}}^{-\pi 2^{-j}} t\left|\widehat{\varphi_{j 0}}(t)\right|^{2} e^{-i k t 2^{j}} d t+\frac{i}{2 \pi} \int_{-\pi 2^{-j}}^{\pi 2^{-j}} t\left|\widehat{\varphi_{j 0}}(t)\right|^{2} e^{-i k t 2^{j}} d t \\
& +\frac{i}{2 \pi} \int_{\pi 2^{-j}}^{3 \pi 2^{-j}} t\left|\widehat{\varphi_{j 0}}(t)\right|^{2} e^{-i k t 2^{j}} d t \\
= & \frac{i}{2 \pi} \int_{-3 \pi 2^{-j}}^{3 \pi 2^{-j}} t\left|\widehat{\varphi_{j 0}}(t)\right|^{2} e^{-i k t 2^{j}} d t \\
= & \frac{i}{2 \pi} \int_{\mathbb{R}} t\left|\widehat{\varphi_{j 0}}(t)\right|^{2} e^{-i k t 2^{j}} d t=b_{k} .
\end{aligned}
$$

Now, $\left\|B_{j}\right\|=\sup _{\|f\|=1}\left\|B_{j} f\right\|$ where $\|f\|^{2}=\sum_{k \in \mathbb{Z}}\left|f_{k}\right|^{2}$. Let $F(t)=\sum_{k \in \mathbb{Z}} f_{k} e^{i k t 2^{j}}$ and define $W(t)=\Gamma_{j}(t) F(t)$. We have

$$
W(t)=\sum_{k \in \mathbb{Z}} \omega_{k} e^{i k t 2^{j}} \quad \text { and } \quad \omega_{k}=\sum_{l \in \mathbb{Z}} b_{k-l} f_{l}=\left(B_{j} f\right)_{k} .
$$

Hence

$$
\begin{aligned}
\|\omega\|^{2} & =\sum_{k \in \mathbb{Z}}\left|\omega_{k}\right|^{2}=\frac{1}{2 \pi 2^{-j}} \int_{-\pi 2^{-j}}^{\pi 2^{-j}}|W(t)|^{2} d t \\
& =\frac{1}{2 \pi 2^{-j}} \int_{-\pi 2^{-j}}^{\pi 2^{-j}}\left|\Gamma_{j}(t) F(t)\right|^{2} d t \\
& \leq \sup _{|t| \leq \pi 2^{-j}}\left|\Gamma_{j}(t)\right|^{2} \frac{1}{2 \pi 2^{-j}} \int_{-\pi 2^{-j}}^{\pi 2^{-j}}|F(t)|^{2} d t \\
& =\sup _{|t| \leq \pi 2^{-j}}\left|\Gamma_{j}(t)\right|^{2}\|f\|^{2} .
\end{aligned}
$$

Then

$$
\left\|B_{j}\right\| \leq \sup _{|t| \leq \pi 2^{-j}}\left|\Gamma_{j}(t)\right| .
$$

On the other hand, $\Gamma_{j}$ is an odd function. Hence

$$
\sup _{|t| \leq \pi 2^{-j}}\left|\Gamma_{j}(t)\right|=\sup _{0 \leq t \leq \pi 2^{-j}}\left|\Gamma_{j}(t)\right| .
$$

But, for $0 \leq t \leq \pi 2^{-j}$, we have $t+\pi 2^{-j+1} \geq \pi 2^{-j+1}$ and $t-\pi 2^{-j+1} \leq 0$. Hence

$$
\widehat{\varphi_{j 0}}\left(t+\pi 2^{-j+1}\right)=0 \quad \text { and } \quad\left(t-\pi 2^{-j+1}\right)\left|\widehat{\varphi_{j 0}}\left(t-\pi 2^{-j+1}\right)\right|^{2} \leq 0
$$

for $t \in\left[0, \pi 2^{-j}\right]$. Thus

$$
\begin{aligned}
\sup _{0 \leq t \leq \pi 2^{-j}}\left|\Gamma_{j}(t)\right| & \leq \pi 2^{-j+1} \sup _{0 \leq t \leq \pi 2^{-j}} t|\widehat{\varphi j 0}(t)|^{2} \\
& =\pi 2^{-j+1} \sup _{0 \leq t \leq \pi 2^{-j}}\left(t 2^{j}\right)\left|\widehat{\varphi}\left(2^{j} t\right)\right|^{2} \\
& =\pi 2^{-j+1} \sup _{0 \leq s \leq \pi} s|\widehat{\varphi}(s)|^{2} .
\end{aligned}
$$

By definition of $\widehat{\varphi}$ we have $|\widehat{\varphi}(s)|^{2} \leq 1$ and therefore $s|\widehat{\varphi}(s)|^{2} \leq \pi$ for $0 \leq s \leq \pi$. Then

$$
\sup _{0 \leq t \leq \pi 2^{-j}}\left|\Gamma_{j}(t)\right| \leq \sup _{0 \leq s \leq \pi} \pi 2^{-j+1} s|\widehat{\varphi}(s)|^{2} \leq \pi^{2} 2^{-j+1} .
$$


Thus

$$
\left\|D_{j}(x)\right\|=\frac{1}{K(x)}\left\|B_{j}\right\| \leq \frac{1}{K(x)} \sup _{|t| \leq \pi 2^{-j}}\left|\Gamma_{j}(t)\right| \leq \frac{\pi^{2} 2^{-j+1}}{K(x)},
$$

which completes the proof of the lemma.

Let us now consider the following approximating problem 2 in $V_{j}$ :

$$
\left\{\begin{array}{l}
K(x) u_{x x}(x, t)=P_{j} u_{t}(x, t), \quad t \geq 0, \quad 0<x<1, \\
u(0, \cdot)=P_{j} g, \\
u_{x}(0, \cdot)=0 \\
u(x, t) \in V_{j} .
\end{array}\right.
$$

Its variational formulation is

$$
\left\{\begin{array}{l}
\left\langle K(x) u_{x x}-u_{t}, \varphi_{j k}\right\rangle=0, \\
\left\langle u(0, \cdot), \varphi_{j k}\right\rangle=\left\langle P_{j} g, \varphi_{j k}\right\rangle, \quad\left\langle u_{x}(0, \cdot), \varphi_{j k}\right\rangle=\left\langle 0, \varphi_{j k}\right\rangle, \quad k \in Z,
\end{array}\right.
$$

where $\varphi_{j k}$ is the orthonormal basis of $V_{j}$ given by the scaling function $\varphi$. Consider a solution $u_{j}$ of the approximating problem (2.3) given by $u_{j}(x, t)=$ $\sum_{l \in Z} w_{l}(x) \varphi_{j l}(t)$. Then, we have $\left(u_{j}\right)_{t}(x, t)=\sum_{l \in Z} w_{l}(x) \varphi_{j l}^{\prime}(t)$ and $\left(u_{j}\right)_{x x}(x, t)$ $=\sum_{l \in Z} w_{l}^{\prime \prime}(x) \varphi_{j l}(t)$. Therefore,

$$
K(x)\left(u_{j}\right)_{x x}(x, t)-\left(u_{j}\right)_{t}(x, t)=K(x) \sum_{l \in Z} w_{l}^{\prime \prime}(x) \varphi_{j l}(t)-\sum_{l \in Z} w_{l}(x) \varphi_{j l}^{\prime}(t) .
$$

Hence

$$
\begin{aligned}
& \left\langle K(x)\left(u_{j}\right)_{x x}-\left(u_{j}\right)_{t}, \varphi_{j k}\right\rangle=0 \Longleftrightarrow\left\langle\sum_{l \in Z} K(x) w_{l}^{\prime \prime} \varphi_{j l}-\sum_{l \in Z} w_{l} \varphi_{j l}^{\prime}, \varphi_{j k}\right\rangle=0 \\
& \Longleftrightarrow \sum_{l \in Z} K(x) w_{l}^{\prime \prime}\left\langle\varphi_{j l}, \varphi_{j k}\right\rangle=\sum_{l \in Z} w_{l}\left\langle\varphi_{j l}^{\prime}, \varphi_{j k}\right\rangle \\
& \Longleftrightarrow K(x) w_{k}^{\prime \prime}=\sum_{l \in Z} w_{l}\left\langle\varphi_{j l}^{\prime}, \varphi_{j k}\right\rangle, \quad k \in Z \\
& \Longleftrightarrow \frac{d^{2}}{d x^{2}} w_{k}=\sum_{l \in Z} w_{l} \frac{1}{K(x)}\left\langle\varphi_{j l}^{\prime}, \varphi_{j k}\right\rangle \Longleftrightarrow \frac{d^{2}}{d x^{2}} w_{k}=\sum_{l \in Z} w_{l}\left(D_{j}\right)_{l k}(x),
\end{aligned}
$$

where, as defined before, $\left(D_{j}\right)_{l k}(x)=\frac{1}{K(x)}\left\langle\varphi_{j l}^{\prime}, \varphi_{j k}\right\rangle$. Thus, we get an infinitedimensional system of ordinary differential equations:

$$
\left\{\begin{array}{l}
\frac{d^{2}}{d x^{2}} w=-D_{j}(x) w \\
w(0)=\gamma \\
w^{\prime}(0)=0
\end{array}\right.
$$

where $\gamma$ is given by

$$
P_{j} g=\sum_{z \in Z} \gamma_{z} \varphi_{j z}=\sum_{z \in Z}\left\langle g, \varphi_{j z}\right\rangle \varphi_{j z}
$$

We will consider (1.1) for functions $g \in L^{2}(R)$ such that $\widehat{g}(\cdot) \exp \left(\frac{|\cdot|}{2 \alpha}\right) \in L^{2}(R)$, where $\widehat{g}$ is the Fourier Transform of $g$. The Inverse Fourier Transform of

\footnotetext{
${ }^{2}$ The projection in the first equation of 2.3 is needed because we can have $\varphi \in V_{j}$ with $\varphi^{\prime} \notin V_{j}$ (see [2] page 224]).
} 
$\exp \left(-\frac{\xi^{2}+|\xi|}{2 \alpha}\right)$, for instance, satisfies this condition. Define

$$
f:=\widehat{g}(\cdot) \exp \left(\frac{|\cdot|}{2 \alpha}\right) \in L^{2}(R)
$$

Theorem 2. Let $u$ be a solution of problem (1.1) with condition $u(0, \cdot)=g$ and let $f$ be given by (2.5). Let $v_{j-1}$ be a solution of problem (2.3) in $V_{j-1}$ with boundary $\widetilde{g}$ such that $\|g-\widetilde{g}\|_{L^{2}(R)} \leq \epsilon$. If $j=j(\epsilon)$ is such that $2^{-j+1}=\frac{\alpha}{\pi^{2}} \log \epsilon^{-1}$, then

$$
\left\|P_{j} v_{j-1}(x, \cdot)-u(x, \cdot)\right\|_{L^{2}(R)} \leq \epsilon^{1-x^{2}}+\|f\|_{L^{2}(R)} \epsilon^{\frac{1}{3}\left(1-x^{2}\right)} .
$$

Proof. See Theorem 3.7 in [2, page 223].

The infinite-dimensional system of ordinary differential equations (2.4) can be written in the following way:

$$
\left\{\begin{array} { l } 
{ \frac { d v } { d x } = - D _ { j } ( x ) w + 0 v , } \\
{ \frac { d w } { d x } = 0 w + v , } \\
{ w ( 0 ) = \gamma \text { and } v ( 0 ) = 0 }
\end{array} \quad \left\{\begin{array}{l}
\frac{d V}{d x}=A_{j}(x) V \\
V(0)=(0, \gamma)^{T}
\end{array}\right.\right.
$$

where $V=(v, w) \in X:=l^{2}(R) \times l^{2}(R), \quad x \in[0,1)$ and

$$
A_{j}(x)=\left[\begin{array}{cc}
0 & -D_{j}(x) \\
1 & 0
\end{array}\right]
$$

with $\left\|A_{j}(x) V\right\|_{X}=\left\|\left(-D_{j}(x) w, v\right)\right\|_{X}=\sqrt{\left\|D_{j}(x) w\right\|_{l^{2}}^{2}+\|v\|_{l^{2}}^{2}}$.

Lemma 3. For all $j \in Z, A_{j}(x): X \longrightarrow X$ is a uniformly bounded linear operator on $x \in[0,1)$.

Proof. By Lemma 1 and the hypothesis $0<\alpha \leq K(x)<+\infty$, we have

$$
\left\|D_{j}(x)\right\| \leq \frac{\pi^{2} 2^{-j+1}}{K(x)} \leq \frac{\pi^{2} 2^{-j+1}}{\alpha}:=K_{j} .
$$

If $\|V\|_{X}=1$, then $\|w\|_{l^{2}} \leq 1$ and $\|v\|_{l^{2}} \leq 1$. So,

$$
\left\|A_{j}(x) V\right\|_{X}=\sqrt{\left\|D_{j}(x) w\right\|_{l^{2}}^{2}+\|v\|_{l^{2}}^{2}} \leq \sqrt{K_{j}^{2}+1} .
$$

Thus, the operator $A_{j}(x)$ is uniformly bounded on $x \in[0,1)$.

Lemma 4. If $\frac{1}{K(x)}$ is Lipschitz on $[0,1)$, then $x \longmapsto D_{j}(x)$ is Lipschitz on [0,1), $\forall j \in Z$. Consequently, $x \longmapsto A_{j}(x)$ is Lipschitz on $[0,1)$.

Proof. $D_{j}(x)=\frac{1}{K(x)} B_{j}$, where $\left(B_{j}\right)_{l k}=\left\langle\varphi_{j l}^{\prime}, \varphi_{j k}\right\rangle$. We have $\left\|B_{j}\right\| \leq \pi^{2} 2^{-j+1}$. Then

$$
\left\|D_{j}(x)-D_{j}(y)\right\| \leq\left|\frac{1}{K(x)}-\frac{1}{K(y)}\right| \pi^{2} 2^{-j+1} \leq L_{j}|x-y|
$$

with $L_{j}=L \cdot \pi^{2} 2^{-j+1}$, where $L$ is the Lipschitz constant of $\frac{1}{K(x)}$.

Now,

$$
\begin{aligned}
\left\|A_{j}(x)-A_{j}(y)\right\| & =\sup _{V \in X,\|V\|=1}\left\|\left(A_{j}(x)-A_{j}(y)\right) V\right\|_{X} \\
& =\sup _{w \in l^{2},\|w\|=1}\left\|\left(D_{j}(x)-D_{j}(y)\right) w\right\|_{l^{2}} \\
& =\left\|D_{j}(x)-D_{j}(y)\right\| \\
& \leq L_{j}|x-y| .
\end{aligned}
$$


Lemma 5. For each $j \in Z$, the operator $[0,1) \ni x \longmapsto A_{j}(x)$ is continuous in uniform operator topology.

Proof. Let $x \in[0,1)$ and $\epsilon>0$. By Lemma $4, A_{j}(x)$ is Lipschitz with Lipschitz constant $L_{j}$. Let $\delta_{\epsilon}:=\epsilon / L_{j}$. We have, for $y \in[0,1)$,

$$
|x-y|<\delta_{\epsilon} \Longrightarrow\left\|A_{j}(x)-A_{j}(y)\right\| \leq L_{j}|x-y|<L_{j} \cdot \delta_{\epsilon}=\epsilon .
$$

By the previous lemmas, we have:

Theorem 6. The infinite-dimensional system of ordinary differential equations (2.4) has a unique solution.

Proof. The above lemmas allow us to apply Theorem 5.1 in [3, page 127] to state this thesis.

Theorem 7. Let $u$ be a solution of problem (1.1) with condition $u(0, \cdot)=g$ where $g$ satisfies (2.5). Then, for any sequence $j_{n}$, such that $j_{n} \longrightarrow-\infty$ as $n \longrightarrow+\infty$, there exists a unique sequence $u_{j_{n}}$ of solutions of the approximating problems (2.3) in $V_{j_{n}}$ with conditions $u_{j_{n}}(0, \cdot)=P_{j_{n}} g$ and $\forall x \in[0,1)$ such that

$$
P_{j_{n}+1} u_{j_{n}}(x, \cdot) \longrightarrow u(x, \cdot) \text { in } L^{2} .
$$

Proof. From Theorem 6 each approximating problem has a unique solution. Then the result follows from Theorem 2 , with $\widetilde{g}=g$, since that $j$ and $\epsilon$ are functionally related by $2^{-j+1}=\frac{\alpha}{\pi^{2}} \log \epsilon^{-1}$ independently of $u$.

Corollary 8. Problem (1.1) has at most one solution, for each $x \in[0,1)$, where $g$ satisfies (2.5).

\section{Conclusion}

We have considered solutions $u(x, \cdot) \in H^{1}(R)$ for the problem $K(x) u_{x x}=u_{t}$, $0<x<1, t \geq 0$, with boundary conditions $g \in L^{2}(R)$ and $u_{x}(0, \cdot)=0$, where $K(x)$ is bounded below by a positive constant, $\frac{1}{K(x)}$ is Lipschitz and $\widehat{g}(\cdot) \exp \left(\frac{|\cdot|}{2 \alpha}\right) \in$ $L^{2}(R)$. We have shown that if a solution exists, it is unique in the sense discussed in the Introduction.

\section{REFERENCES}

1. I. Daubechies, Ten lectures on wavelets, CBMS-NSF Regional Conference Series in Applied Mathematics, 61, SIAM, Philadelphia, PA, 1992. MR.1162107|(93e:42045)

2. J. R. L. de Mattos and E. P. Lopes, A wavelet Galerkin method applied to partial differential equations with variable coefficients, Electronic Journal of Differential Equations Conference 10 (2003), 211-225. MR1976644 (2004e:65113)

3. A. Pazy, Semigroups of linear operators and applications to partial differential equations, Applied Mathematical Sciences, 44, Springer-Verlag, New York, 1983. MR710486 (85g:47061)

Institute of Mathematics, Tecnology Center, Bloco C and Coppe, Systems and Computing Engineering Program, Tecnology Center, Bloco H, Federal University of Rio de Janeiro, Ilha do Fundão, Rio de Janeiro, RJ, CEP 21945-970, Brazil

E-mail address: lopes@cos.ufrj.br

Department of Geometry, Institute of Mathematics, Fluminense Federal University, Rua Mário Santos Braga s/n, Valonguinho, Niterói, Rio de Janeiro, RJ, CeP 24020-140, BRAZIL

E-mail address: jrlinhares@vm.uff.br 